\title{
Prevalence and Risk Factors of Child Malnutrition in Community Based Nutrition Program Implementing and Nonimplementing Districts from South East Amhara, Ethiopia
}

\author{
Behailu Tariku1, Afework Mulugeta'2, Mache Tsadik², Girma Azene ${ }^{3}$ \\ ${ }^{1}$ Health Information Technology Department, Debre Birhan Health Science College, Debre Birhan, Ethiopia \\ ${ }^{2}$ College of Health Sciences, Department of Public Health, Mekelle University, Mekelle, Ethiopia \\ ${ }^{3}$ Tulane University, Addis Abeba, Ethiopia \\ Email: minastariku@gmail.com, Afework.mulugeta@gmail.com, machetasdik@yahoo.com, \\ girmachere@yahoo.com
}

Received 2 April 2014; revised 21 May 2014; accepted 1 June 2014

Copyright (C) 2014 by authors and OALib.

This work is licensed under the Creative Commons Attribution International License (CC BY). http://creativecommons.org/licenses/by/4.0/

\section{Open Access}

\section{Abstract}

Background: The burden of malnutrition in Ethiopia is the second highest in sub Saharan Africa. According to the recently released Ethiopian Demographic and Health Survey report of 2011, the prevalence of child stunting, underweight and wasting were $44 \%$ stunted, $10 \%$ wasted and $29 \%$ underweight respectively. Objectives: To determine the magnitude and risk factors of malnutrition among 6 - 59 months children from Community Based Nutrition Program implementing and nonimplementing districts from south east Amhara. Methodology: Descriptive community based, cross sectional study in which a total of 987 children (620 from Community Based Nutrition implementing and 367 from nonimplementing districts) were sampled. A structured questionnaire was administered to mothers or caregivers of the children. Anthropometric measurements were taken following standard procedures. Nutritional status of the children was determined based on Z scores of $<-2$ or $>=-2$ Standard Deviation. Logistic regression was used to test significant associations between outcome and factor variables. Setting: Rural communities from Artuma Fursi and Kemissie districts. Results: A total of 987 children of 6 - 59 months of age and 987 mothers/caregivers had participated in the study, giving a response rate of $99 \%$. The percentage of male and female children in CBNP district was $330(53.2 \%)$ and $290(46.8 \%)$ and that of non Community Based Nutrition Program (CBNP) district was 192 (52.3\%) \& 175 (47.7\%) respectively. The prevalence of stunting, underweight and wasting were $60.6 \%, 31.1 \%, 12.6 \%$ and $39.0 \%, 27.5 \%, 14.7 \%$ in CBNP and nonCBNP implementing districts, respectively. The possible risk factors for child 
malnutrition were area specific and different for the two districts. Conclusion: Child under nutrition is a serious public health problem in both districts, so that holistic programs need to be designed and implemented according to the guidelines.

\section{Keywords}

Anthropometrics, Community Based Nutrition Program, Stunting, Underweight and Wasting

\section{Introduction}

Protein energy malnutrition (PEM) is one of the most serious nutritional problems with fatal consequences accounting for almost half of all premature deaths from nutrition related diseases [1]. Malnutrition is not only an urgent global health issue; it is also an impediment to productivity, economic growth and poverty eradication [2]. The level of under nutrition and the finding that $90 \%$ of the chronically malnourished (stunted) are to be found in children living in Asia and Africa remain not only unacceptable but also poses a serious challenge against making rapid progress towards improvement in child health and well being. A number of African and Asian countries have wasting rates that exceed 15 per cent, including Bangladesh (17\%), India (20\%) and Sudan (16\%) [3].

The burden of malnutrition in Ethiopia is the second highest in sub Saharan Africa [4]. According to the recently released Ethiopian Demographic and Health Survey [EDHS 2011] report, the prevalence of child stunting, underweight and wasting were $44 \%$ stunted, $10 \%$ wasted and $29 \%$ underweight. When this data is further disaggregated by region, the prevalence of stunting in Amhara region was second to none (52\%) [5]. Besides, according to the World Vision Ethiopia report, in 2000 in Peasant Associations of Artuma Jille and Dawa Cheffa Districts [Kemissie Area Development Program]; the level of wasting have been 8\%, underweight 36\% and stunting 38.7\% [6]. A study conducted in Gumbrit, North West Ethiopia on under five children revealed that 24\% (stunted), 28.5\% (underweight) and 17.7\% (wasted) [7]. A study done in Somalia, Ethiopia showed that the level of wasting, stunting and underweight all increased with age, particularly from the age of 6 - 12 months old. At this age illness is at its highest rate, weaning foods have either not started or are of inadequate quality and mothers start to leave the children for extended periods with other careers. Catch up growth is inadequate thereafter although levels of wasting improve after about 24 months [8]. Similarly, study conducted in Tigray, Northern Ethiopia revealed that older children were more likely to be malnourished [9]. A cross-sectional study conducted on 622 mother-child pairs of 0 - 59 months old children in Mecha and Wenberma districts of West Gojam Zone in 2006 revealed that child's age was among the contributing factors for malnutrition [10]. Study conducted in Somali, Ethiopia revealed that boys tend to be more malnourished than girls at most ages, although this is not understood to be related to any differences in practices or conditions but rather to biological differences. At the same times, stunting is less prevalent in girls than boys (OR 0.76, $\mathrm{p}=0.004)$ although for wasting there is little difference [8]. Likewise, study conducted by Ethiopian Development Research Institute and Save the Children UK showed that being a male child increases the likelihood of being wasted, stunted and underweight [11]. But study conducted in northern Ethiopia contradicts these findings and revealed that there is no statistically significant difference between the HAZ, WAZ and WHZ scores for male and female children [9]. Research conducted in Ethiopia showed that in households where the mother attended more antenatal care visits, the children are less likely to be wasted, and those children receive vaccination against measles are also less likely to be underweight [11]. Another key association that was identified to exist between child morbidity and mother's health care use were both stunting and wasting. There were high rates of diarrhea (23\%) fever (28\%) and cough (32\%) in children. The odds ratio (OR) for wasting and diarrhea was 1.6 and for wasting and fever was 1.7 in a multiple logistical regression model [8].

As long as so many children remain malnourished, Ethiopia will find it difficult to achieve the first Millennium Development Goal which is eradication of extreme poverty and hunger [4]. Indeed, for evidence-based decision making process; timely, adequate and quality of data is mandatory. But the magnitude and the specific risk factors of child malnutrition in the study areas are not well understood. To the best of our knowledge, there are no other studies conducted that adequately quantified the magnitude and risk factors of child malnutrition from the study communities. Therefore, the purpose of this study was to generate a baseline data and assess the 
magnitude and risk factors of malnutrition among children of 6 - 59 months old from CBN implementing and CBN nonimplementing districts from Amhara region.

\section{Methods and Materials}

Community based cross-sectional study was conducted in Artuma Fursi and Kemissie districts of Oromia Zone, South East Amhara, Ethiopia from February to March 2012. Chefa Robit is the capital town of Artuma Fursi district and is located $305 \mathrm{Km}$ away from Addis Abeba. The total population of Artuma Fursi is estimated to be 90, 220 [Male $=44,208$ and Female $=46,012$ ] in 2011 [12]. Out of these, 12,324 are estimated to be children under the age of five. Administratively, the district is composed of 25 [2 urban and 23 rural] kebeles. The health system comprised of public owned 6 health centers and 24 health posts. Community based nutrition program has been introduced in the $3^{\text {rd }}$ quarters of 2009.

Kemissie district is the capital of Oromia zone and is located at $280 \mathrm{Km}$ away from Addis Abeba. The population of Kemissie is estimated to be 52,523 [Male $=25,737$ and Female $=26,786$ ] in 2011 [12]. Out of these 7116 are expected to be children of under the age of five. Kemissie district is composed of 7 [3 urban and 4 rural] kebeles. There are about 20 health institutions in the district. Among these, 4 are government owned comprising of one health centre and 3 health posts. As opposed to the former district, there is no Community Based Nutrition Program in this district [12].

A representative sample size was estimated for this study using a formula for cross sectional study via Epi_Info ${ }^{\mathrm{TM}}$ software version 6. Taking $\mathrm{P}_{1}=$ Prevalence of malnutrition in Kemissie district [38\%] (5), $\mathrm{P}_{2}=$ prevalence of malnutrition in Artuma Fursi district [52\%] [4], ratio of unexposed children to exposed children for CBNP $=(7116 / 12,324=1.7)$, ten percent $(10 \%)$ of any dropout from the study subjects during the study period and a design effect of 2 (two) for the multistage nature of the sampling technique. Finally, a total sample size of 995 of which 625 from Artuma Fursi and 370 from Kemissie district were selected.

Of the five administrative districts of Oromia zone, Artuma Fursi was purposefully selected to represent the CBNP implementation areas whilst Kemissie district was also purposefully selected among the two nonCBNP implementing districts in the Oromia zone of the Amhara Regional State. Simple multistage cluster sampling technique was used. The primary sampling units [PSU] were selected from 23 rural kebeles of Artuma Fursi and 4 rural kebeles of Kemissie districts. Accordingly, five kebeles from Artuma Fursi and 3 kebeles from Kemissie districts were randomly selected as primary sampling units.

Secondary sampling units [SSU], that is, households having children between 6 - 59 months of age were selected by using random sampling techniques. Thus, the numbers of children of 6 - 59 months of age were selected from each selected kebeles. Accordingly, 80 children from Kelo Kebenawa and 80 from Edo Medine, 174 children from Hara, 98 from Fati Komboro, 194 from Bakeja, 165 from Jaraniyo, 118 from Kachur and 86 children from Bilacha kebele were drawn randomly.

In cases where there was more than one child in the households, only one child was randomly selected and considered as study subject. To avoid behavioral contamination of CBN program, one kebele [Kello $=\phi \lambda^{\circ}$ ] was excluded from the study, which is situated between Artuma Fursi and Kemissie districts.

\subsection{Data Collection}

Anthropometric methods (measurements of weight, height and MUAC) and structured questionnaires were used to determine the nutritional status of the children. A total of 14 data collectors comprising 4 clinical nurses, 8 health extension workers and 2 supervisors proficient in the local language were deployed to collect data using pre-tested and structured questionnaires. The study was approved by the Institutional Review Board (IRB) of the College of Health Sciences of Mekelle University. Before the start of data collection, an oral consent was obtained from the care takers.

\subsection{Anthropometric Measurements}

Weight measurement was taken after children were undressed (no shoe, dresses and wet hat). For a child who stands on the weighing scale calmly, the measurement was taken in the nearest $0.1 \mathrm{~kg}$. In the time of refuse to be scaled, children's mother carried and stood on the scale. Finally, the child actual weight was registered by subtracting mother's weight from mother and child weight. 
Recumbent length was taken with measuring board for those children from 6 months to two years of age, while standing height was taken for children two and above years in centimeters to the nearest $1 \mathrm{~cm}$. The age of a child was determined using one of the following: birth certificate, immunization cards or by asking the mother (parents) and if she doesn't remember the exact date, by asking her to determine as close as possible, relating to local events or holidays.

Prior to the actual data collection, a one day intensive training was given to data collectors focusing on the purpose and objectives of the study, survey instruments, administration of the structured questionnaire, anthropometric measurements and ethical considerations. Pre-test on the validity and factual consistencies of the questionnaire and other survey instruments was conducted on $5 \%$ of total sample size in the same area that were later excluded from the main survey. Besides, calibration of the weighing instrument was done against zero reading when weighing every child. Data validity and reliability was maintained through close supervision by the principal investigator and trained supervisor. To minimize systematic error, weight and height of the children were taken three times by the same person and the average value was taken for final analysis.

\subsection{Data Coding and Analysis}

Data were cleaned, coded and entered in to ENA software for anthropometric data and SPSS.version16 software for validation and data analysis. Descriptive statistics such as frequency, percentage, ratio, median, standard error, standard deviation were calculated for dependent variables. For data presentation purposes, frequency distribution tables, graphs and figures were used. Independent-samples t-test was used to compare means of two independent samples. Generalized linear model (GLM) called logistic regression was used to identify major risk factors for child malnutrition. Parameter coefficients of binary logistic regression were estimated by using Wald statistics. Odds ratio [expo $(\beta)$ ] from logistic regression coefficient indicates the increasing $(\mathrm{OR}>1)$ or decreasing $(\mathrm{OR}<1)$ chance of malnutrition with respect to explanatory variables. Models were built for each study areas to express the condition of stunting, underweight and wasting. Then, model adequacy check was done by goodness of fit (classification table, Hosmer and Lemeshow test). For Hosmer and Lemeshow test, if $p>0.05$, the model was taken as statistically satisfactory.

\section{Results}

\subsection{Socio-Demographic Characteristics}

A total of 987 children of 6 - 59 months of age and 986 mothers/caregivers had participated in the study, giving a response rate of 99\%. The percentage of male and female children in CBNP district was 330 (53.2\%) and 290 (46.8\%) and that of nonCBNP district was 192 (52.3\%) \& 175 (47.7\%) respectively. Three hundred forty one (55\%) of the children from the CBNP district were between 6 - 24 months and the remaining 279 (45\%) were between 24 to 59 months. Similarly, 241 (58.3\%) of the children from the nonCBNP were between 6 to 24 months and the rest 153 (41.7\%) were between 24 and 59 months. Five hundred forty one (87.3\%) mother's/ caregiver's of the children in CBNP implementing district were illiterate and only $12.7 \%$ of them had formal education. Regarding educational level of mothers from the nonCBNP implementing district, $80.7 \%$ were illiterate and the remaining $19.3 \%$ had formal education. For those household leaders who were able to list their monthly family income, $86.8 \%$ in CBNP and $69.2 \%$ in nonCBNP districts of the households have income of less than 525 ETB per month which is the lowest government salary.

\subsection{Descriptive Statistics of Study Subjects for Selected Variables}

The mean $( \pm \mathrm{SD}$ ) of HAZ, WAZ, WHZ scores and MUAC in CBNP district were $-2.43 \pm 1.51,-1.34 \pm 1.37$, $-0.01 \pm 1.77$ and $132.7 \pm 12.37$ whereas in nonCBNP district were $-1.34 \pm 2.13,-1.09 \pm 1.36,-0.48 \pm 1.72$ and $134.67 \pm 12.59$ respectively. The major findings of this study revealed that stunting, underweight and wasting in CBNP district were $60.6 \%, 31.1 \%$ and $12.6 \%$, respectively. Contrary, in nonCBNP district stunting, underweight and wasting in nonCBNP district were $39.0 \%, 27.5 \%$ and $14.7 \%$, respectively (Table 1 ).

\subsection{Risk Factors for Child Malnutrition}

\subsubsection{Child Characteristics}

Eighty eight percent of children from the CBNP district and 94\% from nonCBNP district had completed their 
Table 1. Comparison of global, moderate and severe malnutrition among CBNP implementing and non-implementing districts, February 2012.

\begin{tabular}{|c|c|c|c|c|c|c|}
\hline \multirow{2}{*}{ Malnutrition status } & \multicolumn{3}{|c|}{$\begin{array}{l}\text { CBNP implementing district [Artuma Fursi] } \\
\qquad(\mathrm{n}=620)\end{array}$} & \multicolumn{3}{|c|}{$\begin{array}{l}\text { NonCBNP implementing district [Kemissie] } \\
\qquad(\mathrm{n}=367)\end{array}$} \\
\hline & Stunting & Underweight & Wasting & Stunting & Underweight & Wasting \\
\hline Global malnutrition & $376(60.6 \%)$ & $193(31.1 \%)$ & 78 (12.6\%) & $143(39.0 \%)$ & 101 (27.5\%) & $54(14.7 \%)$ \\
\hline Moderate malnutrition & 155 (25\%) & $113(18.2 \%)$ & 49 (7.9\%) & 65 (17.7\%) & $78(21.2 \%)$ & $31(8.4 \%)$ \\
\hline Severe malnutrition & $221(35.6 \%)$ & $80(12.9 \%)$ & $29(4.7 \%)$ & 78 (21.3\%) & $23(6.3 \%)$ & 23 (6.3\%) \\
\hline
\end{tabular}

immunization. But, $87 \%$ of infants in CBNP district and $88 \%$ of infants in nonCBNP district were vaccinated against measles. Eighty five percent of children in CBNP district took vitamin "A" supplementation in the past 6 months prior to data collection. Whereas in nonCBNP implementing district 95\% children took vitamin A supplementation in the past 6 months. Regarding deworming services, 21\% (in CBNP district) and only 7.9\% (in nonCBNP district) of children had taken albendazole/mebendazole tablets during the past six months (Table 2). The prevalence of infectious diseases, in the past two weeks prior to data collection, in CBNP district were 162 (26\%) for diarrhea, 41 (6.6\%) for pneumonia, $28(4.5 \%)$ for malaria and 7 (1.12\%) for measles. Whereas the prevalence in nonCBNP district were: 24 (6.5\%), 27 (7.4\%), 17 (4.6\%) and 1 (0.3\%) for diarrhea, pneumonia, malaria and measles respectively (Table 3). Three hundred seventeen (51.13\%) and 146 (39.89\%) of mothers from CBNP and nonCBNP districts initiated breastfeeding within the first 24 hrs, respectively. Similarly, 54.9\% and $69.8 \%$ of mothers from CBNP and nonCBNP districts exclusively breast fed their infants for four to six months, respectively (Table 3).

\subsubsection{Household and Community Characteristics}

Comparison of the contraceptive utilization practices showed that higher proportions of mothers from the nonCBNP (58\%) took modern contraceptives than mothers from CBNP (21.9\%). Institutional delivery was very low in both districts. A significant proportion of mothers from CBNP (94.2\%) and nonCBNP (87\%) districts delivered at home (Table 3). Out of 620 respondents in CBNP district, 363 (58.5\%) of the households had traditional pit latrine, of which 131 (36\%) of latrines had hand washing facilities and 61 (46.5\%) these latrines had hand washing facilities with soap or ash. On the other hand, in the nonCBNP district, 213 (58.2\%) of the households had traditional pit latrine, of which 94 (44\%) of the latrines had hand washing facilities and of which 68 (72.3\%) had soap/ash used for hand washing after defecation. Regarding drinking water supply, the respondents replied that $31 \%$ in CBNP district and $40 \%$ in nonCBNP district had water from protected source. Significant amounts (69\% in CBNP and 60\% in nonCBNP districts) of the households fetch water from unprotected sources.

In the CBNP district, 316 (50.9\%) children were weighted during growth monitoring session, 329 (53\%) of mothers or care givers participated in community conversation cession, and 265 (42\%) of the respondents had awareness about malnutrition and able to mention at least one sign of malnutrition. On the other hands, in nonCBNP implementing district, the magnitudes were 157 (42.9\%), 70 (19.1\%) and 257 (70.2) for growth monitoring and promotion, community conversation and had awareness about malnutrition and able to mention at least one sign of malnutrition respectively (Table 3).

\subsection{Bivariate and Multivariate Analysis of Logistic Regression}

Bivariate analysis in CBNP district showed that stunting was significantly associated with sex of child [COR = 2.03 at $95 \%$ CI $(1.462,2.813)]$, immunization status of children [COR $=0.465$ at $95 \%$ CI $(0.266,0.813)]$, exclusive breasting feeding [COR $=0.320$ at $95 \%$ CI $(0.151,0.677)$ ], availability of pit latrine [COR $=0.599$ at $95 \%$ CI $(0.429,0.835)]$ and drinking water supply [COR $=0.699$ at $95 \%$ CI $(0.496,0.984)]$. Whereas bivariate analysis of nonCBNP implementing district showed that factors such as; antenatal care service [COR $=1.715$ at $95 \%$ CI $(1.037,2.838)]$, presence of domestic animals [COR $=1.747$ at $95 \%$ CI $(1.010,3.021)]$ and mothers' knowledge towards malnutrition [COR $=1.968$ at $95 \% \mathrm{CI}(1.214,3.190)$ ] were significantly associated with stunting. Multivariate analysis of logistic regression showed a significant association with sex of a child [AOR $=2.357$ at 95\% CI $(1.656,3.354)]$, ANC visit [AOR $=0.605$ at 95\% CI $(0.383,0.953)]$, immunization status of children [AOR $=0.487$ at $95 \%$ CI $(0.267,0.888)]$, exclusive breast feeding [AOR $=0.281$ at $95 \% \mathrm{CI}(0.124,0.636)]$, vi- 
tamin "A" supplementation [AOR $=1.774$ at $95 \%$ CI $(1.066,2.951)]$, availability of pit latrine $[\mathrm{AOR}=0.589$ at $95 \%$ CI $(0.404,0.857)]$, children birth order [AOR $=3.669$ at $95 \%$ CI $(1.299,10.362)]$ and protected water source $[\mathrm{AOR}=0.614$ at $95 \% \mathrm{CI}(0.398,0.949)]$ with stunting in CBNP district. In the case of nonCBNP district multivariate analysis showed that factors like; ANC visit [AOR $=1.839$ at $95 \%$ CI $(1.082,3.126)]$, presence of domestic animals [AOR $=1.859$ at $95 \%$ CI $(1.015,3.403)$, mothers' knowledge towards malnutrition [AOR = 2.226 at $95 \%$ CI $(1.332,3.721)]$ and diarrheal disease [AOR $=2.94$ at $95 \%$ CI $(1.171,7.381)]$ were significantly associated (Table 4).

Table 2. Comparison of child characteristics among CBNP implementing and non-implementing districts, February 2012.

\begin{tabular}{|c|c|c|c|c|}
\hline \multirow{2}{*}{ Risk factors } & \multicolumn{2}{|c|}{$\begin{array}{l}\text { CBNP implementing woreda [AFWHO] } \\
\qquad(\mathrm{n}=620)\end{array}$} & \multicolumn{2}{|c|}{$\begin{array}{l}\text { Non-CBNP implementing woreda [KWAHO] } \\
\qquad(\mathrm{n}=367)\end{array}$} \\
\hline & Magnitude & Percent [\%] & Magnitude & Percent [\%] \\
\hline \multicolumn{5}{|c|}{ Immunization service per schedule } \\
\hline - Yes & 547 & 88.23 & 345 & 94 \\
\hline - No & 73 & 11.77 & 22 & 6 \\
\hline \multicolumn{5}{|l|}{ Measles immunization } \\
\hline - Vaccinated & 545 & 87.9 & 326 & 88.8 \\
\hline - Not vaccinated & 75 & 12.1 & 41 & 11.2 \\
\hline \multicolumn{5}{|c|}{ Vitamin "A" status in the past 6 months } \\
\hline - Yes & 532 & 85.81 & 348 & 94.8 \\
\hline - No & 88 & 14.19 & 19 & 5.2 \\
\hline \multicolumn{5}{|c|}{ Deworming status in the past 6 months } \\
\hline - Yes & 137 & 22.1 & 29 & 7.9 \\
\hline - No & 483 & 77.9 & 338 & 92.1 \\
\hline \multicolumn{5}{|c|}{ Growth monitoring [weighing] } \\
\hline - Yes & 315 & 50.81 & 157 & 42.8 \\
\hline - No & 305 & 49.19 & 210 & 57.2 \\
\hline \multicolumn{5}{|c|}{ Diarrhoea in the last 2 weeks } \\
\hline - Yes & 162 & 26.13 & 24 & 6.5 \\
\hline - No & 458 & 73.87 & 343 & 93.5 \\
\hline \multicolumn{5}{|c|}{ Pneumonia in the last 2 weeks } \\
\hline - Yes & 41 & 6.61 & 27 & 7.4 \\
\hline - No & 579 & 93.39 & 340 & 92.6 \\
\hline \multicolumn{5}{|c|}{ Measles in the past 2 weeks } \\
\hline - Yes & 7 & 1.13 & 1 & 0.3 \\
\hline - No & 613 & 98.87 & 366 & 99.7 \\
\hline \multicolumn{5}{|c|}{ Malaria in the past 2 weeks } \\
\hline - Yes & 28 & 4.52 & 17 & 4.6 \\
\hline - No & 592 & 95.48 & 350 & 95.4 \\
\hline \multicolumn{5}{|l|}{ Exclusive breast feeding } \\
\hline ExcBF for $<4$ months & 53 & 8.62 & 14 & 3.8 \\
\hline ExcBF for 4 to 6 month & 338 & 54.96 & 256 & 69.8 \\
\hline ExcBF for 6 to 9 month & 167 & 27.15 & 61 & 16.6 \\
\hline EXCBF IOr > 9 montns & 57 & 9.27 & 36 & 9.8 \\
\hline \multicolumn{5}{|c|}{ Middle upper arm circumference } \\
\hline MUAC < $115 \mathrm{~mm}$ & 67 & 10.8 & 33 & 9 \\
\hline MUAC > $115 \mathrm{~mm}$ & 553 & 89.2 & 334 & 91 \\
\hline
\end{tabular}


Table 3. Comparison of household and community characteristics among CBNP implementing and nonimplementing districts, February 2012.

\begin{tabular}{|c|c|c|c|c|}
\hline \multirow{2}{*}{ Risk factors } & \multicolumn{2}{|c|}{ CBNP implementing district $(n=620)$} & \multicolumn{2}{|c|}{ Non-CBNP implementing $(\mathrm{n}=367)$} \\
\hline & Magnitude & Percent [\%] & Magnitude & Percent [\%] \\
\hline \multicolumn{5}{|l|}{ Birth control method } \\
\hline - Yes & 136 & 21.93 & 250 & 58.1 \\
\hline - No & 484 & 78.07 & 117 & 41.9 \\
\hline \multicolumn{5}{|l|}{ Postnatal care } \\
\hline - Yes & 202 & 32.58 & 299 & 81.5 \\
\hline - $\quad$ No & 418 & 67.42 & 68 & 18.5 \\
\hline \multicolumn{5}{|c|}{ Parents' participation in GM } \\
\hline - Yes & 174 & 28.29 & 36 & 9.84 \\
\hline - No & 441 & 71.71 & 330 & 90.16 \\
\hline \multicolumn{5}{|c|}{ Practice of community conversation } \\
\hline - Yes & 329 & 53.06 & 70 & 19.1 \\
\hline - $\quad$ No & 291 & 46.94 & 297 & 80.9 \\
\hline \multicolumn{5}{|l|}{ Malnutrition knowledge } \\
\hline - Yes & 265 & 42.74 & 257 & 70 \\
\hline - $\quad$ No & 355 & 57.26 & 110 & 30 \\
\hline \multicolumn{5}{|l|}{ Water sources } \\
\hline - Protected & 199 & 32.1 & 151 & 41.2 \\
\hline - Unprotected & 421 & 67.9 & 216 & 58.8 \\
\hline \multicolumn{5}{|l|}{ Presence of latrine } \\
\hline - Present & 363 & 58.55 & 213 & 58 \\
\hline - Not present & 257 & 41.45 & 154 & 42 \\
\hline \multicolumn{5}{|l|}{ Round trip to fetch water } \\
\hline Less than 30 minutes & 194 & 31.29 & 212 & 57.8 \\
\hline 30 to 60 minutes & 320 & 51.61 & 129 & 35.1 \\
\hline$>60$ minutes & 106 & 17.1 & 26 & 7.1 \\
\hline
\end{tabular}

Significant associations of child wasting were observed with age of children [AOR $=1.33$ at 95\% CI (1.064, $2.821)$ ], postnatal care service [AOR $=0.480$ at $95 \% \mathrm{CI}(0.266,0.864)]$ and availability of pit latrine $[\mathrm{AOR}=$ 0.599 at $95 \% \mathrm{CI}(0.368,0.975)]$ in CBNP implementing district during bivariate and multivariate analysis. In the same fashion, age of the children $[\mathrm{AOR}=5.786$ at $95 \% \mathrm{CI}(2.870,11.663)]$ and mothers' knowledge towards malnutrition $[\mathrm{AOR}=0.398$ at $95 \% \mathrm{CI}(0.208,0.763)]$ were significantly associated with wasting in nonCBNP implementing district during bivariate and multivariate analysis (Table 5).

There were significant association between antenatal care visit [AOR $=1.837$ at $95 \% \mathrm{CI}(1.115,3.025)]$, deworming service [AOR $=1.815$ at $95 \%$ CI $(1.179,2.794)$ ], availability of pit Latrine [AOR $=0.675$ at $95 \%$ CI $(0.466,0.976)]$, diarrheal diseases $[\mathrm{AOR}=1.814$ at $95 \% \mathrm{CI}(1.227,2.681)]$ and presence of bed net $[\mathrm{AOR}=$ 0.540 at $95 \%$ CI $(0.361,0.807)]$ with child underweight both in bivariate and multivariate analysis of logistic regression in CBNP implementing district. Where as in nonCBNP implementing district only availability of pit latrine [COR $=0.620$ at $95 \% \mathrm{CI}(0.390,0.983)]$ is significantly associated with child underweight in bivariate analysis. While factors such as family size [AOR $=3.406$ at $95 \%$ CI $(1.162,9.983)$ ], availability of pit latrine [AOR $=0.543$ at $95 \%$ CI $(0.325,0.909)]$ and child birth order $[\mathrm{AOR}=0.273$ at $95 \%$ CI $(0.084,0.887)]$ were significantly associated with child under weight during multivariate logistic regression analysis (Table 6).

Comparison of child malnutrition in study districts showed that stunting and underweight is significantly higher in CBNP implementing district than nonCBNP district. Whereas wasted children are slightly but not significantly higher in nonCBNP implementing district than CBNP district (Table 7). 
Table 4. Bivariate and multivariable analysis of factors associated with child stunting in CBNP and nonCBNP implementing districts from Oromia Zone, South East Amhara, Ethiopia, 2012.

\begin{tabular}{|c|c|c|c|c|c|c|c|c|}
\hline \multirow{3}{*}{ Variables } & \multicolumn{8}{|c|}{ Stunting (variables were selected at a p value of 0.15 using backward elimination) } \\
\hline & \multicolumn{4}{|c|}{ CBN district $(n=620)$} & \multicolumn{4}{|c|}{ Non CBN district $(n=367)$} \\
\hline & No & Yes & COR $(95 \% \mathrm{CI})$ & AOR (95\%CI) & No, $n(\%)$ & Yes, $n(\%)$ & COR (95\%CI) & AOR $(95 \% \mathrm{CI})$ \\
\hline \multicolumn{9}{|l|}{ Head of HH } \\
\hline Father & $\begin{array}{c}218 \\
(35.15)\end{array}$ & $349(56.3)$ & 1 & & $198(54.0)$ & $138(37.6)$ & $\begin{array}{c}3.624 \\
(1.358,9.670)^{*}\end{array}$ & $\begin{array}{c}4.257 \\
(1.500,12.083)^{*}\end{array}$ \\
\hline Mother & $26(4.25)$ & $27(4.35)$ & $0.637(0.12,3.3)$ & & $26(7.1)$ & $5(1.4)$ & 1 & 1 \\
\hline \multicolumn{9}{|l|}{ Family size } \\
\hline$<=4$ & $64(10.3)$ & $91(14.7)$ & $\begin{array}{c}0.899 \\
(0.577,1.399)\end{array}$ & $\begin{array}{c}0.327 \\
(0.129,0.826)^{*}\end{array}$ & $62(16.9)$ & $33(9.0)$ & $\begin{array}{c}1.107 \\
(0.585,2.093)\end{array}$ & $\begin{array}{c}1.822 \\
(0.905,3.668)\end{array}$ \\
\hline $5-7$ & 113 (18.2) & 179 (28.9) & $\begin{array}{c}1.001 \\
(0.681,1.473)\end{array}$ & $\begin{array}{c}0.507 \\
(0.241,1.066)\end{array}$ & $110(30)$ & $85(23.2)$ & $\begin{array}{c}1.607 \\
(0.923,2.799)\end{array}$ & $\begin{array}{c}1,572 \\
(0.878,2.812)\end{array}$ \\
\hline$>=8$ & $67(10.8)$ & 106 (17.1) & 1 & 1 & $52(14.2)$ & $25(6.8)$ & 1 & 1 \\
\hline \multicolumn{9}{|l|}{ Sex } \\
\hline Male & 104 (16.8) & $226(36.5)$ & $\begin{array}{c}2.028 \\
(1.462,2.813)\end{array}$ & $\begin{array}{c}2.357 \\
(1.656,3.354)^{*}\end{array}$ & 113 (30.8) & 79 (21.6) & $1.2(0.79,1.8)$ & \\
\hline Female & $140(22.6)$ & $150(24.2)$ & 1 & 1 & $111(30.2)$ & $64(17.4)$ & 1 & \\
\hline \multicolumn{9}{|l|}{ Age } \\
\hline$<23.9$ & 111(17.9) & 146 (23.6) & $\begin{array}{c}0.761 \\
(0.549,1.054)\end{array}$ & $\begin{array}{c}0.711 \\
(0.497,1.018)\end{array}$ & 101 (27.5) & 51 (13.9) & $\begin{array}{c}0.675 \\
(0.438,1.040)\end{array}$ & $\begin{array}{c}0.635 \\
(0.400,1.006)\end{array}$ \\
\hline $24-59.9$ & 133 (21.5) & 230 (37.1) & 1 & 1 & 123 (33.5) & $92(25.1)$ & 1 & 1 \\
\hline \multicolumn{9}{|l|}{ ANC visits } \\
\hline$<4$ & 206 (33.2) & 295 (47.6) & $\begin{array}{c}0.672 \\
(0.439,1.027)\end{array}$ & $\begin{array}{c}0.605 \\
(0.383,0.953)^{*}\end{array}$ & $158(43.1)$ & 115 (31.3) & $\begin{array}{c}1.715 \\
(1.037,2.837)^{*}\end{array}$ & $\begin{array}{c}1.839 \\
(1.082,3.126)^{*}\end{array}$ \\
\hline$>=4$ & $38(6.1)$ & 81(13.1) & 1 & 1 & $66(18.0)$ & $28(7.6)$ & 1 & 1 \\
\hline \multicolumn{9}{|l|}{$\begin{array}{l}\text { Domestic } \\
\text { animals }\end{array}$} \\
\hline Yes & $\begin{array}{c}179 \\
(28.87)\end{array}$ & $\begin{array}{c}252 \\
(40.64)\end{array}$ & 1 & & 170 (46.3) & $121(33.0)$ & $\begin{array}{c}1.747 \\
(1.010,3.021)^{*}\end{array}$ & $\begin{array}{c}1.859 \\
(1.015,3.403)^{*}\end{array}$ \\
\hline No & $65(10.5)$ & $124(20)$ & $0.72(0.52,1.05)$ & & $54(14.7)$ & $22(6.0)$ & 1 & 1 \\
\hline \multicolumn{9}{|l|}{$\begin{array}{l}\text { Colustrum } \\
\text { feeding }\end{array}$} \\
\hline Yes & 113 (18.3) & 204 (33.0) & $\begin{array}{c}1.003 \\
(0.328,3.065)\end{array}$ & $\begin{array}{c}1.305 \\
(0.382,4.457)\end{array}$ & 95 (25.9) & 53 (14.5) & $\begin{array}{c}0.86 \\
(0.45,1.53)\end{array}$ & \\
\hline No & $126(20.4)$ & $161(26.1)$ & $\begin{array}{c}0.710 \\
(0.232,2.171)\end{array}$ & $\begin{array}{c}0.757 \\
(0.226,2.539)\end{array}$ & $86(24)$ & $62(16.9)$ & $\begin{array}{c}1.1 \\
(0.62,1.97)\end{array}$ & \\
\hline $\begin{array}{c}\text { I don't } \\
\text { remember }\end{array}$ & $5(0.8)$ & $9(1.5)$ & 1 & 1 & $43(11.7)$ & $28(7.6)$ & 1 & \\
\hline \multicolumn{9}{|l|}{ Immunization } \\
\hline Yes & $226(36.5)$ & 321 (51.8) & $\begin{array}{c}0.465 \\
(0.266,0.813)^{*}\end{array}$ & $\begin{array}{c}0.487 \\
(0.267,0.888)^{*}\end{array}$ & 214 (58.3) & $131(35.7)$ & $\begin{array}{c}1.96 \\
(0.82,4.66)\end{array}$ & \\
\hline No & $18(2.9)$ & 55 (8.9) & 1 & 1 & $10(2.7)$ & 12 (3.3) & 1 & \\
\hline \multicolumn{9}{|l|}{ EBF } \\
\hline 0 - 3 months & $6(1)$ & $9(1.5)$ & $\begin{array}{c}0.511 \\
(0.157,1.659)\end{array}$ & $\begin{array}{c}0.402 \\
(0.114,1.414)\end{array}$ & $9(2.45)$ & $5(1.4)$ & $\begin{array}{c}0.69 \\
(0.19,2.48)\end{array}$ & \\
\hline 4 - 5 months & $33(5.3)$ & $31(5.0)$ & $\begin{array}{c}0.320 \\
(0.151,0.677)^{*}\end{array}$ & $\begin{array}{c}0.281 \\
(0.124,0.636)^{*}\end{array}$ & 161 (43.9) & 95 (25.9) & $\begin{array}{c}0.74 \\
(0.36,1.49)\end{array}$ & \\
\hline 6 - 8 months & 189 (30.5) & 289 (46.6) & $\begin{array}{c}0.521 \\
(0.287,0.945)^{*}\end{array}$ & $\begin{array}{c}0.406 \\
(0.213,0.773)^{*}\end{array}$ & $34(9.2)$ & $27(7.3)$ & $\begin{array}{c}0.99 \\
(0.43,2.27)\end{array}$ & \\
\hline $\begin{array}{l}9 \text { and above } \\
\text { months }\end{array}$ & $16(2.6)$ & $47(7.6)$ & 1 & 1 & 20 & 16 & 1 & \\
\hline
\end{tabular}




\section{Continued}

\begin{tabular}{|c|c|c|c|c|c|c|c|c|}
\hline VAS & & & & & & & & \\
\hline Yes & 205 (33.1) & 327 (52.7) & $\begin{array}{c}1.270 \\
(0.805,2.002)\end{array}$ & $\begin{array}{c}1.774 \\
(1.066,2.951)^{*}\end{array}$ & 214 (58.3) & 134 (36.5) & $\begin{array}{c}0.69 \\
(0.27,1.75)\end{array}$ & \\
\hline No & $39(6.3)$ & $49(7.9)$ & 1 & 1 & $10(2.7)$ & $9(2.5)$ & 1 & \\
\hline \multicolumn{9}{|l|}{ Measles sickness } \\
\hline Yes & $5(0.8)$ & $2(0.3)$ & $\begin{array}{c}0.256 \\
(0.049,1.328)\end{array}$ & $\begin{array}{c}0.162 \\
(0.021,1.262)\end{array}$ & $1(0.27)$ & $0(0.0)$ & $0.0(0.0,0.0)$ & \\
\hline No & 239 (38.6) & 374 (60.3) & 1 & 1 & $\begin{array}{c}223 \\
(60.73)\end{array}$ & 143 (39) & 1 & \\
\hline \multicolumn{9}{|l|}{ Latrine } \\
\hline Yes & $161(26.0)$ & 202 (32.6) & $\begin{array}{c}0.599 \\
(0.429,0.835)^{*}\end{array}$ & $\begin{array}{c}0.589 \\
(0.404,0.857)^{*}\end{array}$ & $134(36.5)$ & 79 (21.5) & $\begin{array}{c}0.83 \\
(0.54,1.27)\end{array}$ & \\
\hline No & 83 (13.4) & $174(28.1)$ & 1 & & $90(24.5)$ & $64(17.5)$ & 1 & \\
\hline \multicolumn{9}{|l|}{$\begin{array}{c}\text { Protected water } \\
\text { source }\end{array}$} \\
\hline Yes & 90 (14.5) & 109 (17.6) & $\begin{array}{c}0.699 \\
(0.496,0.984)^{*}\end{array}$ & $\begin{array}{c}0.614 \\
(0.398,0.949)^{*}\end{array}$ & 85 (23.2) & 65 (17.7) & $\begin{array}{c}1.36 \\
(0.89,2.08)\end{array}$ & \\
\hline No & $154(24.8)$ & $267(43.1)$ & 1 & & 139 (37.9) & 78 (21.2) & 1 & \\
\hline \multicolumn{9}{|l|}{ Deworming } \\
\hline Yes & $47(7.6)$ & $90(14.5)$ & 1 & & $14(3.8)$ & $15(4.1)$ & $\begin{array}{c}1.758 \\
(0.821,3.762)\end{array}$ & $\begin{array}{c}1.836 \\
(0.810,4.161)\end{array}$ \\
\hline No & 197 (31.8) & $286(46.1)$ & $0.76(0.51,1.13)$ & & $210(57.2)$ & 128 (34.9) & 1 & 1 \\
\hline \multicolumn{9}{|l|}{ Birth order } \\
\hline First & $34(5.5)$ & $52(8.4)$ & $\begin{array}{c}1.147 \\
(0.670,1.963)\end{array}$ & $\begin{array}{c}3.669 \\
(1.299,10.362)^{*}\end{array}$ & 41 (11.2) & $17(4.6)$ & $\begin{array}{c}0.71 \\
(0.34,1.48)\end{array}$ & \\
\hline Second and third & 70 (11.3) & $129(20.8)$ & $\begin{array}{c}1.382 \\
(0.897,2.129)\end{array}$ & $\begin{array}{c}2.601 \\
(1.112,6.085)^{*}\end{array}$ & 78 (21.3) & $52(14.2)$ & $\begin{array}{c}1.13 \\
(0.63,2.05)\end{array}$ & \\
\hline Fourth and fifth & 74 (11.9) & 107 (17.3) & $\begin{array}{c}1.084 \\
(0.702,1.676)\end{array}$ & $\begin{array}{c}1.704 \\
(0.786,3.696)\end{array}$ & $59(16)$ & 47 (12.8) & $\begin{array}{c}1.36 \\
(0.74,2.49)\end{array}$ & \\
\hline Six and above & 66 (10.7) & $88(14.2)$ & 1 & & 46 (12.5) & 27 (7.3) & 1 & \\
\hline \multicolumn{9}{|l|}{$\begin{array}{c}\text { Knowledge } \\
\text { about } \\
\text { malnutrition }\end{array}$} \\
\hline Yes & 111 (17.9) & $154(24.8)$ & 1 & & 145 (39.5) & $112(30.5)$ & $\begin{array}{c}1.968 \\
(1.214,3.190)^{*}\end{array}$ & $\begin{array}{c}2.226 \\
(1.332,3.721)^{*}\end{array}$ \\
\hline No & 133 (21.5) & $222(35.8)$ & $1.2(0.87,1.67)$ & & 79 (21.5) & $31(8.5)$ & 1 & 1 \\
\hline \multicolumn{9}{|l|}{ Child diarrhea } \\
\hline Yes & $67(10.8)$ & 95 (15.3) & $0.89(0.62,1.28)$ & & $10(2.7)$ & $14(3.8)$ & $\begin{array}{c}2.322 \\
(1.002,5.381)\end{array}$ & $\begin{array}{c}2.940 \\
(1.171,7.381)^{*}\end{array}$ \\
\hline No & 177 (28.6) & $281(45.3)$ & 1 & & $214(58.3)$ & $129(35.2)$ & 1 & 1 \\
\hline \multicolumn{9}{|l|}{$\begin{array}{c}\text { Presence of bed } \\
\text { net }\end{array}$} \\
\hline Yes & $175(28.2)$ & $255(41.1)$ & 1 & & 194 (52.9) & $114(31.1)$ & $\begin{array}{c}0.608 \\
(0.347,1.065)\end{array}$ & $\begin{array}{c}0.586 \\
(0.321,1.071)\end{array}$ \\
\hline No & 69 (11.1) & $121(19.6)$ & $1.2(0.85,1.71)$ & & $30(8.2)$ & 29 (7.9) & 1 & 1 \\
\hline \multicolumn{9}{|l|}{ Monthly income } \\
\hline Lowest income & 210 & 328 & $1.25(0.33,4.7)$ & & $155(42.2)$ & $99(27)$ & $1.15(0.51,2.59)$ & \\
\hline Medium income & 30 & 43 & $1.15(0.28,4.6)$ & & 51 (13.9) & $34(9.3)$ & $1.2(0.49,2.91)$ & \\
\hline Highest income & 4 & 5 & 1 & & $18(4.9)$ & $10(2.7)$ & 1 & \\
\hline
\end{tabular}


Table 5. Bivariate and multivariable analysis of factors associated with child wasting in CBNP and nonCBNP implementing districts from Oromia Zone, South East Amhara, Ethiopia, 2012.

\begin{tabular}{|c|c|c|c|c|c|c|c|c|}
\hline \multirow{3}{*}{ Variables } & \multicolumn{8}{|c|}{ Wasting (variables were selected at a p value of 0.15 using backward elimination) } \\
\hline & \multicolumn{4}{|c|}{ CBN district $(n=620)$} & \multicolumn{4}{|c|}{ Non CBN district $(n=367)$} \\
\hline & No & Yes & COR $(95 \% \mathrm{CI})$ & AOR (95\%CI) & No, $n(\%)$ & Yes, $n(\%)$ & COR $(95 \% \mathrm{CI})$ & AOR (95\%CI) \\
\hline \multicolumn{9}{|l|}{ Marital status } \\
\hline Married & $491(79.2)$ & $73(11.8)$ & $0.89(0.2,4.0)$ & & $292(79.6)$ & $53(14.4)$ & $\begin{array}{c}3.812 \\
(0.502,28.944)\end{array}$ & $\begin{array}{c}4.435 \\
(0.531,37.028)\end{array}$ \\
\hline $\begin{array}{l}\text { Others (single, } \\
\text { divorced, } \\
\text { widowed) }\end{array}$ & $51(8.2)$ & $5(0.8)$ & 1 & & $21(5.7)$ & $1(0.3)$ & 1 & 1 \\
\hline \multicolumn{9}{|l|}{ Sex } \\
\hline Male & 295 (47.6) & $35(5.7)$ & $\begin{array}{c}0.682 \\
(0.423,1.098)\end{array}$ & $\begin{array}{c}0.636 \\
(0.389,1.039)\end{array}$ & $165(45)$ & 27 (7.35) & $0.89(0.5,1.6)$ & \\
\hline Female & 247 (39.8) & $43(6.9)$ & 1 & 1 & $148(40.3)$ & 27 (7.35) & 1 & \\
\hline \multicolumn{9}{|l|}{ Age } \\
\hline$<23.9$ & 215 (34.7) & $42(6.8)$ & $\begin{array}{c}1.774 \\
(1.101,2.860)^{*}\end{array}$ & $\begin{array}{c}1.733 \\
(1.064,2.821)\end{array}$ & $111(30.3)$ & $41(11.2)$ & $\begin{array}{c}5.739 \\
(2.950,11.165)\end{array}$ & $\begin{array}{c}5.786 \\
(2.870,11.663)\end{array}$ \\
\hline $24-59.9$ & 327 (52.7) & $36(5.8)$ & 1 & 1 & $202(55.0)$ & $13(3.5)$ & 1 & 1 \\
\hline \multicolumn{9}{|l|}{ PNC service } \\
\hline Yes & $186(30.2)$ & $16(2.6)$ & $\begin{array}{c}0.499 \\
(0.280,0.890)^{*}\end{array}$ & $\begin{array}{c}0.480 \\
(0.266,0.864)\end{array}$ & 252 (68.7) & 47 (12.8) & $1.6(0.7,3.77)$ & \\
\hline No & 354 (57.4) & $61(9.9)$ & 1 & 1 & $61(16.6)$ & $7(1.9)$ & 1 & \\
\hline \multicolumn{9}{|l|}{$\begin{array}{c}\text { Latrine } \\
\text { availability }\end{array}$} \\
\hline Yes & 327 (52.7) & $36(5.8)$ & $\begin{array}{c}0.564 \\
(0.350,0.908)^{*}\end{array}$ & $\begin{array}{c}0.599 \\
(0.368,0.975)\end{array}$ & $\begin{array}{c}184 \\
(50.15)\end{array}$ & $29(7.9)$ & $\begin{array}{c}0.81 \\
(0.45,1.45)\end{array}$ & \\
\hline No & $215(34.7)$ & $42(6.8)$ & 1 & 1 & $\begin{array}{c}129 \\
(35.15)\end{array}$ & $25(6.8)$ & 1 & \\
\hline \multicolumn{9}{|l|}{$\begin{array}{l}\text { Colustrum } \\
\text { feeding }\end{array}$} \\
\hline Yes & $280(45.2)$ & 37 (5.9) & $2.06(0.55,7.74)$ & & 125 (34.1) & $23(6.3)$ & $\begin{array}{c}2.428 \\
(0.883,6.681)\end{array}$ & $\begin{array}{c}2.868 \\
(0.980,8.394)\end{array}$ \\
\hline No & $250(40.3)$ & 37 (5.9) & $1.84(0.49,6.91)$ & & $122(33.2)$ & $26(7.1)$ & $\begin{array}{c}2.813 \\
(1.032,7.66)^{*}\end{array}$ & $\begin{array}{c}2.747 \\
(0.951,7.933)\end{array}$ \\
\hline $\begin{array}{l}\text { I don't } \\
\text { remember }\end{array}$ & $11(1.8)$ & $3(0.5)$ & 1 & & $66(18.0)$ & $5(1.4)$ & 1 & 1 \\
\hline \multicolumn{9}{|l|}{$\begin{array}{l}\text { Knowledge } \\
\text { about child } \\
\text { malnutrition }\end{array}$} \\
\hline Yes & 234 (37.7) & $31(5)$ & $1.15(0.71,1.87)$ & & 229 (62.4) & $28(7.6)$ & $\begin{array}{c}0.395 \\
(0.219,0.712)^{*}\end{array}$ & $\begin{array}{c}0.398 \\
(0.208,0.763)^{*}\end{array}$ \\
\hline No & 308 (49.6) & $47(7.7)$ & 1 & & 84 (22.9) & $26(7.1)$ & 1 & 1 \\
\hline \multicolumn{9}{|l|}{ Practice of CC } \\
\hline Yes & $298(48.1)$ & $31(5)$ & $1.85(1.14,3.01)^{*}$ & & 68 (18.5) & $2(0.5)$ & $\begin{array}{c}0.139 \\
(0.033,0.584)\end{array}$ & $\begin{array}{c}0.139 \\
(0.032,0.604)^{*}\end{array}$ \\
\hline No & 244 (39.4) & $47(7.7)$ & 1 & & 245 (66.8) & $52(14.2)$ & 1 & 1 \\
\hline \multicolumn{9}{|l|}{ Child diarrhea } \\
\hline Yes & $142(22.9)$ & $20(3.2)$ & $1.03(0.59,1.77)$ & & $18(4.9)$ & $6(1.6)$ & $\begin{array}{c}2.049 \\
(0.774,5.420)\end{array}$ & $\begin{array}{c}2.087 \\
(0.705,6.179)\end{array}$ \\
\hline No & $400(64.5)$ & $58(9.4)$ & 1 & & $295(80.4)$ & 48 (13.1) & 1 & 1 \\
\hline
\end{tabular}


Table 6. Bivariate and multivariable analysis of factors associated with child underweight in CBNP and nonCBNP implementing districts from Oromia Zone, South East Amhara, Ethiopia , 2012.

\begin{tabular}{|c|c|c|c|c|c|c|c|c|}
\hline \multirow{3}{*}{ Variables } & \multicolumn{8}{|c|}{ Underweight (variables were selected at a p value of 0.15 using backward elimination) } \\
\hline & \multicolumn{4}{|c|}{ CBN district $(n=620)$} & \multicolumn{4}{|c|}{ Non CBN district $(n=367)$} \\
\hline & No, n (\%) & Yes, n (\%) & COR $(95 \% \mathrm{CI})$ & AOR $(95 \% \mathrm{CI})$ & No, n (\%) & Yes, n (\%) & COR $(95 \% \mathrm{CI})$ & AOR (95\%CI) \\
\hline \multicolumn{9}{|l|}{ Head of HH } \\
\hline Father & $387(62.4)$ & $173(27.9)$ & $0.33(0.07,1.51)$ & & $237(64.6)$ & $99(27.0)$ & $\begin{array}{c}6.057 \\
(1.418,25.871)^{*}\end{array}$ & $\begin{array}{c}7.752 \\
(1.717,35.002)\end{array}$ \\
\hline Mother & $40(6.45)$ & $20(3.25)$ & 1 & & $29(7.9)$ & $2(0.54)$ & 1 & 1 \\
\hline Sex & & & & & & & & \\
\hline Male & $224(36.1)$ & 106 (17.1) & $1.1(0.78,1.55)$ & & 145 (39.5) & 47 (12.8) & $\begin{array}{c}0.726 \\
(0.459,1.150)\end{array}$ & $\begin{array}{c}0.659 \\
(0.402,1.081)\end{array}$ \\
\hline Female & 203 (32.7) & $87(14.1)$ & 1 & & $121(33.0)$ & 54 (14.7) & 1 & 1 \\
\hline \multicolumn{9}{|l|}{ Family size } \\
\hline$<=4$ & 110 (17.7) & 45 (7.25) & $0.77(0.48,1.23)$ & & 72 (19.6) & $23(6.3)$ & $\begin{array}{c}1.047 \\
(0.517,2.122)\end{array}$ & $\begin{array}{c}3.406 \\
(1.162,9.983)^{*}\end{array}$ \\
\hline $5-7$ & 204 (32.9) & $88(14.2)$ & $0.81(0.54,1.21)$ & & 135 (36.8) & $60(16.4)$ & $\begin{array}{c}1.457 \\
(0.792,2.679)\end{array}$ & $\begin{array}{c}2.016 \\
(0.913,4.453)\end{array}$ \\
\hline$>=8$ & 113 (18.2) & $60(9.7)$ & 1 & & $59(16.1)$ & $18(4.90)$ & 1 & 1 \\
\hline \multicolumn{9}{|l|}{ ANC visits } \\
\hline$<4$ & 334 (53.9) & 167 (26.9) & $\begin{array}{c}1.788 \\
(1.115,2.870)^{*}\end{array}$ & $\begin{array}{c}1.837 \\
(1.115,3.025)^{*}\end{array}$ & $192(52.3)$ & $81(22.1)$ & $\begin{array}{c}1.561 \\
(0.893,2.727)\end{array}$ & $\begin{array}{c}1.563 \\
(0.868,2.812)\end{array}$ \\
\hline$>=4$ & $93(15.0)$ & $26(4.2)$ & 1 & 1 & $74(20.2)$ & $20(5.5)$ & 1 & 1 \\
\hline \multicolumn{9}{|l|}{$\mathrm{EBF}$} \\
\hline 0 - 3 months & $41(6.6)$ & $12(13.2)$ & $0.58(0.25,1.36)$ & & $1(0.3)$ & $2(0.5)$ & $\begin{array}{c}7.800 \\
(0.641,94.917)\end{array}$ & $\begin{array}{c}12.138 \\
(0.792,185.932)\end{array}$ \\
\hline 4 - 5 months & 236 (38) & $102(16.4)$ & $0.86(0.47,1.57)$ & & $21(5.7)$ & $10(2.7)$ & $\begin{array}{c}1.857 \\
(0.667,5.174)\end{array}$ & $\begin{array}{c}2.015 \\
(0.666,6.095)\end{array}$ \\
\hline 6 - 8 months & 109 (17.6) & $58(9.4)$ & $1.06(0.56,2.01)$ & & 205 (55.9) & 79 (21.5) & $\begin{array}{c}1.503 \\
(0.716,3.155)\end{array}$ & $\begin{array}{c}2.311 \\
(1.004,5.322)^{*}\end{array}$ \\
\hline $\begin{array}{l}9 \text { and above } \\
\text { months }\end{array}$ & $38(6.1)$ & $19(3.1)$ & 1 & & 39 (10.6) & $10(2.7)$ & 1 & 1 \\
\hline \multicolumn{9}{|l|}{ Deworming } \\
\hline Yes & 84 (13.6) & $53(8.6)$ & $\begin{array}{c}1.546 \\
(1.04,2.297)^{*}\end{array}$ & $\begin{array}{c}1.815 \\
(1.179,2.794)^{*}\end{array}$ & $19(5.2)$ & $10(2.7)$ & $\begin{array}{c}1.429 \\
(0.640,3.188)\end{array}$ & $\begin{array}{c}2.106 \\
(0.863,5.143)\end{array}$ \\
\hline No & 343 (55.3) & $140(22.6)$ & 1 & 1 & 247 (67.3) & 91 (24.8) & 1 & 1 \\
\hline \multicolumn{9}{|l|}{$\begin{array}{c}\text { Latrine } \\
\text { availability }\end{array}$} \\
\hline Yes & $268(43.2)$ & 95 (15.3) & $\begin{array}{c}0.575 \\
(0.408,0.811)^{*}\end{array}$ & $\begin{array}{c}0.675 \\
(0.466,0.978)^{*}\end{array}$ & $163(44.4)$ & 50 (13.6) & $\begin{array}{c}0.620 \\
(0.390,0.983)^{*}\end{array}$ & $\begin{array}{c}0.543 \\
(0.325,0.909)^{*}\end{array}$ \\
\hline No & 159 (25.7) & 98 (15.8) & 1 & 1 & $103(28.1)$ & 51 (13.9) & 1 & 1 \\
\hline \multicolumn{9}{|l|}{$\begin{array}{l}\text { Child } \\
\text { Diarrhea }\end{array}$} \\
\hline Yes & 96 (15.5) & 66 (10.7) & $\begin{array}{c}1.792 \\
(1.232,2.605)\end{array}$ & $\begin{array}{c}1.814 \\
(1.227,2.681)^{*}\end{array}$ & $14(3.8)$ & $10(2.7)$ & $\begin{array}{c}1.979 \\
(0.849,4.612)\end{array}$ & $\begin{array}{c}2.170 \\
(0.868,5.427)\end{array}$ \\
\hline No & 331 (53.4) & 127 (20.5) & 1 & 1 & 252 (68.7) & $91(24.8)$ & 1 & 1 \\
\hline \multicolumn{9}{|l|}{$\begin{array}{l}\text { Presence of } \\
\text { bed net }\end{array}$} \\
\hline Yes & 315 (50.8) & 115 (18.6) & $\begin{array}{c}0.524 \\
(0.366,0.751)^{*}\end{array}$ & $\begin{array}{c}0.540 \\
(0.361,0.807)^{*}\end{array}$ & $228(62.1)$ & $80(21.8)$ & $0.64(0.35,1.15)$ & \\
\hline No & $112(18.1)$ & 78 (12.6) & 1 & 1 & 38 (10.4) & $21(5.7)$ & 1 & \\
\hline $\begin{array}{l}\text { Community } \\
\text { conversation }\end{array}$ & & & & & & & & \\
\hline
\end{tabular}




\begin{tabular}{|c|c|c|c|c|c|c|c|}
\hline \multicolumn{8}{|l|}{ Continued } \\
\hline Yes & 239 (38.5) & 90 (14.5) & $\begin{array}{c}0.68 \\
(0.49,0.97)^{*}\end{array}$ & 57 (15.5) & 13 (3.5) & $\begin{array}{c}0.542 \\
(0.282,1.040)\end{array}$ & $\begin{array}{c}0.558 \\
(0.276,1.128)\end{array}$ \\
\hline No & $188(30.3)$ & 103 (16.7) & 1 & $209(57.0)$ & $88(24.0)$ & 1 & 1 \\
\hline \multicolumn{8}{|l|}{ Birth order } \\
\hline First & 58 (9.35) & $28(4.5)$ & $0.95(0.54,1.66)$ & 46 (12.5) & $12(3.3)$ & $\begin{array}{c}0.692 \\
(0.305,1.566)\end{array}$ & $\begin{array}{c}0.273 \\
(0.084,0.887)^{*}\end{array}$ \\
\hline $\begin{array}{l}\text { Second and } \\
\text { third }\end{array}$ & $141(22.7)$ & 58 (9.35) & $0.81(0.51,1.27)$ & $96(26.2)$ & 34 (9.3) & $\begin{array}{c}0.939 \\
(0.492,1.791)\end{array}$ & $\begin{array}{c}0.510 \\
(0.208,1.251)\end{array}$ \\
\hline $\begin{array}{l}\text { Fourth and } \\
\text { fifth }\end{array}$ & $126(20.3)$ & $55(8.9)$ & $0.85(0.54,1.36)$ & 71 (19.4) & 35 (9.5) & $\begin{array}{c}1.306 \\
(0.679,2.514)\end{array}$ & $\begin{array}{c}0.821 \\
(0.364,1.849)\end{array}$ \\
\hline Six and above & $\begin{array}{c}102 \\
(16.45)\end{array}$ & $52(8.4)$ & 1 & 53 (14.4) & $20(5.5)$ & 1 & 1 \\
\hline
\end{tabular}

Table 7. Comparison of nutritional status of children from the two districts (CBN and Non CBN), Oromia Zone, South East Amhara, Ethiopia, 2012.

\begin{tabular}{cccccccccc}
\hline \multirow{2}{*}{ Variable } & \multicolumn{3}{c}{ Stunting } & \multicolumn{3}{c}{ Underweight } & \multicolumn{3}{c}{ Wasting } \\
\cline { 2 - 11 } & No & Yes & COR & No & Yes & COR & No & Yes & COR \\
\hline Districts & & & & & & & & & \\
& & & & & & & & & \\
CBN & $244(24.7)$ & $376(38.1)$ & $(1.853,3.145)$ & $427(43.3)$ & $193(19.6)$ & $(0.895,1.583)$ & $542(54.9)$ & $78(7.9)$ & $(0.574,1.212)$ \\
Non CBN & $224(22.7)$ & $143(14.5)$ & 1 & $266(27.0)$ & $101(10.2)$ & 1 & $313(31.7)$ & $54(5.5)$ & 1 \\
\hline
\end{tabular}

Analysis of Independent t-test of the two groups that is, districts undergoing CBN program and yet not implemented one, revealed that there are statistical difference between the means of average monthly income of the mothers/caregivers, distance traveled by the mothers/caregivers to fetch drinking water, average height of the children and average height to age $\mathrm{Z}$ score of the children at $\mathrm{p}$ value less than 0.05 (Table 8).

\section{Discussion}

The prevalence of stunting and underweight in CBNP district are critical problems as per the WHO classification of severity of malnutrition [13]; but the problems in nonCBNP district is series. When we observe the magnitude of severe acute malnutrition, it is a series problem in both study districts. The comparison showed that the prevalence of stunted children in CBNP district is significantly higher than that of nonCBNP district. This could be as a result of different factors. Some of them can be seen from independent $t$ test analysis (Table 8). As you had seen from the result part, factors affecting the prevalence of acute and chronic malnutrition can be illustrated as follows:

Sex is an important demographic variable in nutritional survey and this study revealed that child's sex is one of the determinant factors for stunted children in CBNP implementing district but not in nonCBNP district. Cross-sectional studies conducted in different parts of the world [14]-[17] have shown that boys are at higher risk of stunting than girls. Some study conducted in India and Brazil describe that the great vulnerability of boys over girls at this age is a yet not well understood phenomenon [18] [19]. Despite, this may leads to an argument that sex preference may be practiced by mothers in community based nutrition implementing district than nonCBNP district. The differences could be attributed to unmeasured factors such as parental care-giving behaviors which might need further investigation. To the contrary, studies conducted in India and Ethiopia [20] [21] showed that girls are at higher risk of malnutrition than boys. In addition to these, study conduct in Ethiopia, Guatamala and Gaza strip, showed that [10] [22] [23] there is no gender difference in the nutritional status between boys and girls; this is the same as in nonCBN implementing district. This study suggests that in CBN and nonCBN districts sex related differences in malnutrition associated with child caring behaviour requires further investigations.

Since immunisation protects susceptible children from communicable diseases, children from the CBNP implementing district were less likely to be affected by stunting than non-immunised children. This could be due to the fact that immunisation reduces child morbidity from vaccine preventable communicable diseases which may 
Table 8. T test analysis of selected variables of CBNP implementing and not yet implemented districts from South East Amhara, Ethiopia, 2012.

\begin{tabular}{|c|c|c|c|c|}
\hline Variable & $\mathrm{CBN}$ & NCBN & $\mathrm{t}$ & $\mathrm{P}$ \\
\hline Average monthly income & 318 & 418 & -7.68 & $<0.0001$ \\
\hline Mean age & 26.4 & 26.7 & -0.32 & 0.75 \\
\hline Average weight & 1.49 & 1.57 & -2.44 & 0.0147 \\
\hline Average walking time to fetch water & 50.9 & 35.5 & 8.22 & $<0.0001$ \\
\hline Average height & 79.3 & 82.8 & -4.87 & $<0.0001$ \\
\hline Average MUAC & 132.8 & 134.7 & -2.30 & 0.0214 \\
\hline Average HAZ & -2.43 & -1.34 & -9.35 & $<0.0001$ \\
\hline Average WAZ & -1.34 & -1.09 & -2.8 & 0.0052 \\
\hline Average WHZ & -0.01 & -0.48 & 4.11 & $<0.0001$ \\
\hline
\end{tabular}

lead a child to malnutrition [24]. Besides, malnutrition is the prime indication for immunisation. Similar study result was observed in Brazil and India where immunization was significantly associated with malnutrition [19] [25]. We observed no significant association between child immunization and stunting in nonCBNP implementing district. This might be due to significant amounts (94.26\%) of children were immunized in nonCBNP implementing district.

Availability of pit latrine was one of the predictors of stunting in CBNP implementing district. Children living in households with traditional pit latrine were less likely to be affected by stunting than children without pit latrine. This indicates that hygiene and sanitation is a key to child survival [26]. This finding is similar with study conducted in Ethiopia, Turkana (Kenya) and Jakarta that children from households that use pit latrine or flush toilet were less likely to be stunted or underweight than non users [16] [27] [28]. But we found no significant association between latrine and stunting in nonCBNP district $(\mathrm{p}>0.05)$.

Infants of 0 - 6 months should fed on only breast milk and shouldn’t given any fluids, milk from animals, any semi solid or liquid food products, even clean water. Since exclusive breast feeding, within the first six months of the child ensures maximum protection against mortality, morbidity and malnutrition. In addition exclusive breastfeeding contribute a lot in child spacing and lower fertility rate. But at the start of the seventh month of child's life breast milk alone cannot meet up a children's energy and nutrient requirements [29]. This study observed that exclusive breast feeding was another risk factor for stunting in CBNP implementing district. Children who were exclusively breast fed for 4 to 6 months were less likely to be stunted than children who were exclusively breast fed for more than 9 months. This could be explained by the fact that breast-feeding is the natural and perfect form of infant feeding for meeting a child's nutritional needs during the first six months of child life. This in turn implies that as the duration of exclusive breast feeding extends from the recommended duration, then the risk of chronic malnutrition increases because of low intake of supplementary food that is rich in energy. This result is similar with researches done in Vietnam [15] and Ethiopia [30]. But, in nonCBNP district, no significant association was observed between exclusive breast feeding and stunting ( $>0.05)$.

Diarrheal disease remains a leading cause of mortality and morbidity of children in Sub-Saharan Africa region where unique geographic, economic, political, socio-cultural, and personal factors interact to create distinctive continuing challenges to its prevention and control [31]. Acute and chronic infections contribute to malnutrition by causing decreased food intake, impaired absorption, increased losses of fluid, electrolytes, protein, and iron, and by altering the normal metabolism [32]. Evidence from numerous studies of children under five years of age in developing countries suggests that both acute and persistent episodes of diarrhea predispose to or exacerbate malnutrition [33]-[35], and conversely chronic malnutrition may be a risk factor for diarrhea [36]. Likewise this study showed that diarrheal disease was a predictor for underweight in CBNP district. Even if the direction of causality is not clear for the association, the study showed that children having history of diarrhea during the past two weeks prior to data collection were more likely to be underweight than non diseased children. Though, it could be assumed that having diarrhoea could not only be a cause but also a consequence of being underweight. The occurrence of diarrhea could be due to the use of unsafe source of water supply and low coverage of pit latrine. This study result was in agreement with the study reported from Brazil in 2009 and Nepal [19] [22]. 
The effect of family size on nutrition outcomes varies according to the dependency nature of family members (Mukherjee and Benson 2003; Garrett and Ruel 1999; Charmarwabagwala et al. 2005). Meaning if the member of families are working age group, by virtue of economics scales, they earn a lot, then improve welfare of the household, then most probably improved nutrition. Contrary in a household if there are many young children, they compete for resource and children of higher birth order most probably become vulnerable to malnutrition [37]. This study depicts that households having more than or greater than eight family sizes are 3 times more vulnerable to stunting in CBNP district. While in nonCBNP district the more family size predisposes the child with underweight. The results of this study are inconsistent with study conducted in the rural areas of North Central and Southern region of Nigeria [37]. The reason could be socio cultural differences between these study areas.

Most countries including Ethiopia give deworming and vitamin A supplementation twice a year to their children of 6 - 59 months of age to protect them from mortality, morbidity and malnutrition. Our study showed that provision of albendazole or mebendazole to the child significantly prevents underweight in CBNP district but not in nonCBNP district (Table 7). Whereas children who took vitamin A supplementation less likely to develop stunting than those children who didn't take vitamin A in CBNP district. This could be due to deworming prevents children from diarrhea, which in turn could probably cause malnutrition. In addition, it prevents loss of micronutrients, particularly iron that contributes to growth retardation [29].

Moreover, age is a predictor variable for acute malnutrition and statistically associated with wasting in study districts. At age of less than 24 months the burden was higher than age greater than 24 months. This might be due to the fact that at this age category, infectious diseases could at its highest rate and aggravated by poor hygiene and sanitation condition of study districts. This might also indicate that there may be problem associated with prolonged exclusive breast-feeding and inappropriate feeding practices in CBNP district. The result of this study compares favorably with the finding from a similar study conducted in Nepal [38]. Contrary to this result, different findings conducted in Somalia and Tigray indicated that the levels of malnutrition increased with age [8] [9].

Monthly income and child malnutrition were not statistically associated $(\mathrm{p}>0.05)$. This could be due to the fact that significant proportions [86\% in CBNP and 69\% in nonCBNP] of the parents were categorized under poor income class [income less than lowest government salary $(<525$ ETB)]. Furthermore, this could be most probably due to community's habit of not telling the exact amount of monthly income [information bias] for the sake of food aid or other aids. A similar result was observed in Somali, Ethiopia in 2002 which revealed that income and child malnutrition were not statistically significant [8]. This finding contradicts studies done in different places that economic status was found to be determinants of child malnutrition [15] [16] [21] [24].

All in all, the program implemented, that is community based nutrition was believed to bring improvements in the status of child malnutrition, but within intervention district there is no significant improvements seen when compared to nonintervention district. Primarily the program was launched to build up communities and families' capacity and ownership to make informed decisions on child care practices at family and community levels, through major implementation approaches including Growth Monitoring and Promotion, supported by Community Conversation. But, most probably due to poor management of the program, it brought no significant changes. But, the study revealed that significantly higher proportion of chronic malnutrition was seen in intervention district than nonintervention district. This result was similar with study conducted in Guatamala, where, child malnutrition was higher in intervention area than comparison area [39].

\section{Strengths and Limitations of the Study}

The use of large sample size, high response rate and use of multivariate analysis to control the effects of confounders and effect modifiers were some of the strengths of this study. Whereas, cross-sectional nature of the data, recall bias (respondent not remembering correctly) and interviewer effects, might affect the internal validity of the results and might be considered as limitations of this study.

\section{Conclusion}

Our results established that child malnutrition (both acute and chronic) is serious public health problem which require strong remedial action in both districts. Besides, the possible risk factors for child malnutrition were area specific calling for different cost effective interventions. 


\section{Recommendations}

\subsection{Recommendations for Artuma Fursi District [CBNP Implementing District]}

To overcome the burden of child malnutrition Community Based Nutrition Program, exclusive breast feeding, IMNCI, maternal health services, hygiene and sanitation activities should be given due emphasis. In addition qualitative study is imperative to assess how and why the significant differences between intervention and non intervention study areas occurred and to identify other contributing factors for child malnutrition.

\subsection{Recommendations for Kemissie District [Non-CBNP Implementing District]}

Community Based Nutrition Program should be launched with due emphasis and good program management. Other services like maternal health services (ANC, delivery and PNC) should be strengthened, and nutrition surveillance for timely screening and treatment of acute malnutrition should be established. Moreover, integrated management of childhood illness and hygiene and sanitation conditions should be strengthened.

\section{Acknowledgements}

The support of many individuals and institutions had made this study possible. We would like to thank; Mekelle University, Artuma Fursi district health office, all study participants, and data collectors for their all kinds of support and love they have given to us.

\section{References}

[1] World Health Organization (2000) Turning the tides of Malnutrition; Responding to the Challenges of 21st Century. CH-1211, WHO, Geneva.

[2] Addis Continental Institute of Public Health (2009) Community-Based Sub-Component of Ethiopian National Nutrition Program Baseline Survey Report. ACIPH, Addis Ababa.

[3] UNICEF (2009) Tracking Progress on Child and Maternal Nutrition. UNICEF, New York.

[4] Mekonnen, A., Jones, N. and Tefera, B. (2005) Tackling Child Malnutrition in Ethiopia: Do the Sustainable Development Poverty Reduction Programme’s Underlying Policy Assumptions Reflect Local Realities? Young Lives, Save the Children UK, Working Paper No.19.

[5] Central Statistical Authority (CSA), Ethiopia (2012) Demographic and Health Survey Report at National Level. CSA, Addis Ababa, Ethiopia, ICF International Calverton, Maryland, USA.

[6] World Vision Ethiopia (2000) Food Security Monitoring Report. WVE Grants Division, Addis Ababa.

[7] Melkie, E. (2007) Assessment of Nutritional Status of Preschool Children of Gumbrit, North West Ethiopia. The Ethiopian Journal of Health Development, 21, 125-129.

[8] Save the Children (2007) The Causes of Malnutrition in Children under 3 in the Somali Region of Ethiopia Related to Household Caring Practices Preliminary Report. Save the Children (UK), Ethiopia.

[9] Mulugeta, A., Hagos, F., Kruseman, G., Linderhof, V., Stoecker, B. and Abraha, Z., et al. (2010) Child Malnutrition in Tigray, Northern Ethiopia. East African Medical Journal, 87, 248-254.

[10] Teshome, B., Kogi-Makau, W., Getahun, Z. and Taye, G. (2009) Magnitude and Determinants of Stunting in Children under Five Years of Age in Food Surplus Region of Ethiopia: The Case of West Gojam Zone. Ethiopian Journal of Health Development, 23, 98-106.

[11] Ethiopian Development Research Institute (Save the Children) (2005) Tackling Child Malnutrition in Ethiopia: To What Extents Do the SDPRP's Underlying Policy Assumptions Reflect Local Realities? Save the Children (UK), Ethiopia.

[12] Oromia Zonal Health Department (2011) Annual Activity Performance Report to Be Sent to Anhara Regional Health Bureau. Kemissie, Ethiopia.

[13] World Health Organization (1997) WHO Global Database on Child Growth and Malnutrition. Program of Nutrition. WHO Document No. WHO/NUT/97.4, WHO, Geneva.

[14] Malla, S. and Shrestha, S.M. (2004) Complementary Feeding Practices and its Impact on Nutritional Status of under Two Old Children in Urban Areas of the Kathmandu, Nepal. Journal of Nepal Health Research Council, 2, 1-4.

[15] Hien, N.N. and Hoa, N.N. (2009) Nutritional Status and Determinants of Malnutrition in Children under Three Years of Age in Nghean, Vietnam. Asian Network for Scientific Information. Pakistan Journal of Nutrition, 8, 958-964. http://dx.doi.org/10.3923/pjn.2009.958.964 
[16] Girma, W. and Genebo, T. (2002) Determinants of Nutritional Status of Women and Children in Ethiopia. Addis Ababa, Ethiopia, Ethiopia Health and Nutrition Research Institute, ORC Macro, Calverton.

[17] Kielmann, N.S., Kielmann, A.A. and Grewal, O.K. (1976) Etiological Determinants of Protein Calorie Undernutrition in a Rural Child Community. Acta Tropica, 33, 342-368.

[18] Levinson, F.J. (1974) Morinda: An Economic Analysis of Malnutrition among Young Children in Rural India. CornellMIT International Nutrition Policy Series, Cambridge.

[19] Sarni, R.O., Carvalho, M.F., de Monte, C.M., Albuquerque, Z.P. and Souza, F.I. (2009) Anthropometric Evaluation, Risk Factors for Malnutrition, and Nutritional Therapy for Children in Teaching Hospitals in Brazil. Jornal de Pediatria, 85, 223-228. http://dx.doi.org/10.1590/S0021-75572009000300007

[20] Rao, G.R., Ladusingh, L. and Pritamjit, R. (2004) Nutritional Status of Children in North-East India. Asia-Pacific Population Journal, 19, 39-56.

[21] Gemechu, A. (2000) Determinants of Nutritional Status of Children in Amhara Region. M.Sc. Thesis in Demography, DTRC/IDR, Addis Ababa University, Addis Ababa.

[22] Valverde, V., MejIa-Pivaral, V., Delgado, H., Belizan, J., Klein, R.E. and Martorell, R. (1981) Income and Growth Retardation in Poor Families with Similar Living Conditions in Rural Guatemala. Ecology of Food and Nutrition, 10, 24l248.

[23] Schoenbaum, M., Tulchinsky, T.H. and Abed, Y. (1995) Gender Differences in Nutritional Status and Feeding Patterns among Infants in the Gaza Strip. American Journal of Public Health, 85, 965-969. http://www.pubmedcentral.nih.gov/articlerender.fcgi?artid=1615541

[24] World Health Organization (1984) Indications and Contraindications for Vaccines Used in the Expanded Programme on Immunization. Bulletin of the World Health Organization, 62, 357-366.

[25] Devadas, R.P., Rajalakshmi, R. and Kaveri, R. (1980) Influence of Family Income and Parent's Education on the Nutritional Status of Pre-School Children. Indian Journal of Nutrition and Dietetics, 17, 237.

[26] UNICEF and WHO (2011) World Water Day: Highlighting the International Year of Sanitation. http://www.worldwaterday.org/

[27] Alderman, H. (2006) Determinants of Malnutrition in Senegal: Individual, Household, Community Variables, and Their Interaction. World Bank PSE Paris and UMR 379, Marseille.

[28] Bardosono, S., Sastroamidjojo, S. and Lukito, W. (2007) Determinants of Child Malnutrition during the 1999 Economic Crisis in Selected Poor Areas of Indonesia. Asia Pacific Journal of Clinical Nutrition, 16, 512-526.

[29] Essential Intervention for Reducing Malnutrition in Infants and Young Children (2008) A Leadership Agenda for Action. The Coalition for Sustainable Nutrition Security in India. September.

[30] Amsalu, S. and Tigabu, Z. (2006) Risk Factors for Severe Acute Malnutrition in Children under the Age of Five: A Case-Control Study. Ethiopian Journal of Health Development, 22, 21-25.

[31] Hamer, H., Simon, F., Thea, D. and Keusch, G.T. (1998) Childhood Diarrhoae in Sub-Saharan Africa. Child Health Research Project Special Report, 2.

[32] Keusch, G.T. and Scrimshaw, N.S. (1986) Selective Primary Health Care: Strategies for Control of Disease in the Developing World. XXIII. Control of Infection to Reduce the Prevalence of Infantile and Childhood Malnutrition. Clinical Infectious Diseases, 8, 273-287. http://dx.doi.org/10.1093/clinids/8.2.273

[33] Marsden, P.D. (1964) The Sukuta Project: A Longitudinal Study of Health in Gambian Children from Birth to 18 Months of Age. Transactions of the Royal Society of Tropical Medicine and Hygiene, 58, 455-488. http://dx.doi.org/10.1016/0035-9203(64)90101-4

[34] Mata, L.J., Kromal, R.A., Urrutia, J.J. and Garcia, B. (1977) Effect of Infection on Food Intake and the Nutritional State: Perspectives as Viewed from the Village. American Journal of Clinical Nutrition, 30, 1215-1227.

[35] Martorell, R., Habicht, J.P., Yarbrough, C., Lechtig, A., Klein, R.E. and Western, K.A. (1975) Acute Morbidity and Physical Growth in Rural Guatamalan Children. American Journal of Diseases of Children, 129, 1296-1301.

[36] Briend, A. (1990) Is Diarrhoea a Major Cause of Malnutrition among Underfives in Developing Countries? A Review of Available Evidence. European Journal of Clinical Nutrition, 44, 611-628.

[37] A Quantitative Analysis of Determinants of Child and Maternal Malnutrition in Nigeria December 2009.

[38] Martorell, R., Leslie, J. and Moock, P.R. (1984) Characteristics and Determinants of Child Nutritional Status in Nepal. American Journal of Clinical Nutrition, 39, 74-86. http://www.ajcn.org

[39] USAID. Evaluation of the Growth Monitoring and Promotion Component of the Integrated Care for Children and Women at the Community Level (AIEPI AINM-C). Calidad en Salud, University Research Co., LLC (URC), Las Margaritas. http://www.urc-chs.com/uploads/resourcefiles/CalidadenSaludAIEPIAINM-CMay08.pdf 


\section{Abbreviation and Acronyms}

\begin{tabular}{ll} 
AF & Artuma Fursi \\
ANC & Antenatal care \\
CBNP & Community Based Nutrition Program \\
CC & Community Conversation \\
CI & Confidence Interval \\
EBF & Exclusive Breast Feeding \\
EDHS & Ethiopian Demographic and Health Survey \\
ETB & Ethiopian Birr \\
GLM & Generalized Linear Model \\
HAZ & Height-for-Age Z Score \\
HEWs & Health Extension Workers \\
HH & House holds \\
NNP & National Nutrition Program \\
OR & Odd Ratio \\
PEM & Protein Energy Malnutrition \\
PNC & Postnatal care \\
PSU & Primary Sampling Unit \\
SPSS & Statistical Package for Social Sciences \\
SSU & secondary Sampling Units \\
UNICEF & United Nation International child and Educational Fund \\
VAS & Vitamine A Supplimentation \\
WAZ & Weight-for-Age Z Score \\
WHO & World Health Organization \\
WHZ & Weight-for-Height Z Score \\
& \\
\hline SH
\end{tabular}

